\title{
COALITION FORMATION GAMES FOR ENERGY-EFFICIENT WIRELESS NETWORK COCAST
}

\author{
Hung-Quoc Lai ${ }^{1,2}$, Yan Chen ${ }^{2}$, and K. J. Ray Liu ${ }^{2}$ \\ ${ }^{1}$ US Army RDECOM CERDEC, RDER-STA-DS, Aberdeen Proving Ground, MD 21005, USA. \\ ${ }^{2}$ Department of Electrical and Computer Engineering,University of Maryland, College Park, MD 20742, USA.
}

\begin{abstract}
Wireless network cocast (WNC) is a cooperative communication protocol that overcomes the issues of imperfect frequency and timing synchronization while achieving spatial diversity to substantially reduce transmit power over direct transmission (DTX). Due to additional processing power in receiving and retransmitting each other information, however, not all nodes and WNC networks result in energy efficiency. To ensure energy efficient WNC networks, a TDMA-based merge process based on coalition formation games is proposed to orderly form cooperative groups. A node is merged into a cooperative group if the merge leads to power saving without causing additional power burden to individual nodes. Simulation shows that WNC networks require much less power to provide comparable quality of service of DTX networks. In addition, WNC networks achieve even power distribution, which improves the network lifetime.
\end{abstract}

Index Terms - Cooperative communications, wireless network cocast, coalition formation games, merge process, energy efficiency.

\section{INTRODUCTION}

Cooperative communications have been attractive due to the ability of improving communication performance [1]. Due to the broadcast nature of wireless communications, nodes in a network can overhear the transmitted information and help relaying it to the intended destination. Cooperative communications thus form a virtual antenna array to combat channel fading through spatial diversity and can dramatically reduce the transmission power in comparison with direct transmission (DTX).

Among many protocols in cooperative communications, wireless network cocast (WNC) provides a framework to eliminate the issue of imperfect frequency and timing synchronization while its associated space-time network codes (STNCs) ensure full spatial diversity [2]. In this paper, we first examine the power consumption of WNC networks and compare with that of DTX networks for a given symbol error rate (SER). The power consumption model considers the processing power at the transmitter and receiver radio-frequency (RF) components and the required transmit power, which accounts for power amplifier (PA) energy efficiency and the peak-to-average-power ratio (PAPR), to convey the information over the medium between the transmitter and the receiver. Computer simulations were conducted and show that depending on the network dimensions, we may not achieve power saving using WNC over DTX. In addition, for WNC networks associated with network power saving, due to the additional processing power in reception and retransmission in cooperation, not all user nodes achieve individual power saving.
To ensure energy efficiency to the network and also individuals, we leverage coalition formation games to propose a merge process for WNC networks. Coalition formation games have been applied in economics and political science. Recently, they are also used to analyze performance of communication networks [3]. The games consider the trade-off between the gain and the cost when forming coalitions. To form cooperative groups, the merge-and-split rules [4] have been used, which are proved to converge to a unique solution with arbitrary merge and split iterations.

In WNC networks, each user node when joining a cooperative group achieves transmit power saving through spatial diversity while incurs additional processing power due to the reception and retransmission of overheard information. The tradeoff represents the gain and the cost in cooperation. Unlike [5], where the authors assume channel information is available at user nodes, we realize that information exchange also require certain medium access control and thus offer a TDMA-based merge process to orderly and efficiently form the cooperative groups in WNC networks. In addition, the complexity of exchanging information in wireless networks is too high, especially with large numbers of user nodes, we propose a heuristic approach in forming cooperative groups. The condition for a merge is that the merge leads to power saving for the group without causing additional power burden for the individual members. Simulation shows that WNC networks require 3.4 times less total power, which comprises the transmit power and processing power, to provide comparable quality of service of DTX networks. In addition, WNC networks achieve even power distribution, which improves the network lifetime, which is defined as the time until the first node dies.

The rest of this paper is organized as follows. After this introduction section, the power consumption model is introduced in Section 2. Simulation of power consumption in WNC and DTX systems for various transmission distances is also presented. In Section 3, the merge process used to form cooperative groups is presented, and simulation illustrating the performance of the proposed merge process is also provided. Lastly, we draw some conclusions in Section 4.

\section{POWER CONSUMPTION IN WNC AND DTX NETWORKS}

\subsection{System Model}

We consider a network consisting of $N$ user nodes denoted as $U_{1}, U_{2}, \ldots, U_{N}$ having their own information that need to be delivered to a common base node $U_{0}$ as shown in Figure 1. All nodes in the network are assumed to have single antennas. The channels are modeled as narrow-band Rayleigh fading with additive white Gaussian noise (AWGN). The chan- 


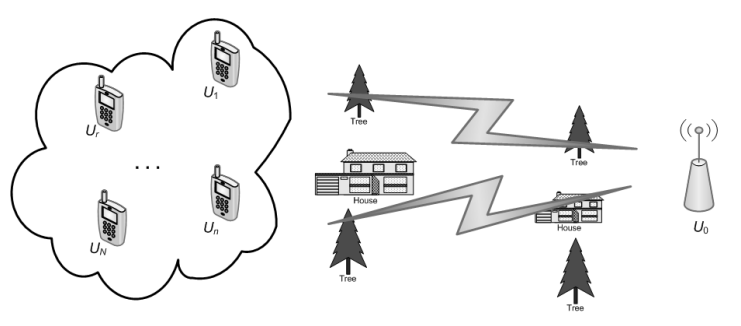

Fig. 1. System model.

nel variance between arbitrary nodes $U_{u}$ and $U_{v}$ is $\sigma_{u v}^{2}=$ $\kappa d_{u v}^{-\alpha}$, where $d_{u v}, \kappa, \alpha$ are the distance between $U_{u}$ and $U_{v}$, the pathloss constant, and the pathloss exponent, respectively. The pathloss constant can be modeled as

$$
\kappa=\left(\frac{\lambda}{4 \pi}\right)^{2} \frac{G_{t} G_{r}}{N_{f}},
$$

where $\lambda$ is the carrier wavelength, $G_{t}$ and $G_{r}$ are, respectively, the transmit and receive antenna gains, and $N_{f}$ is the noise figure.

Two transmission protocols, namely DTX and WNC, are considered for the network. Each user node in DTX directly transmit its own information to the base station while in WNC, the $N$ user nodes cooperate to each other following the multipoint-to-point WNC scheme in [2]. The SER for transmitting information from $U_{n}$ to $U_{0}$ in DTX using phase-shift keying (PSK) modulation can be expressed as [6]

$$
S E R_{n}^{D T X}=F\left(1+\frac{b E_{s, n}^{D T X} \sigma_{0 n}^{2}}{N_{0} \sin ^{2} \theta}\right),
$$

where $b=\sin ^{2}(\pi / \mathcal{M})$ is a coefficient associated with $\mathcal{M}$ PSK modulation, $N_{0}$ is the thermal noise power spectral density (PSD), $E_{s, n}^{D T X}$ is the energy per symbol, and

$$
F(x(\theta))=\frac{1}{\pi} \int_{0}^{(\mathcal{M}-1) \pi / \mathcal{M}} \frac{1}{x(\theta)} d \theta .
$$

The WNC network encompasses two phases, the STP and the RTP [2]. In the first phase, each user node takes turn to transmit its own information to the base node. Due to the broadcast nature of wireless communications, other user nodes overhear the information and help relaying it to the base node in the later phase. In the RTP, each user node acting as a relay node constructs a unique signal in FDMA or CDMA manners. The signal is a combination of the overheard information previously received from the $(N-1)$ source nodes in the STP. The base node $U_{0}$ jointly detects the transmitted information from the received signals in both STP and RTP.

We assume decode-and-forward protocol in cooperation, where a relay node decodes the overheard information and then re-encodes and transmits it to the base node if the decoding is correct. The exact SER expression associated with information from $U_{n}$ for $\mathcal{M}$-PSK modulation is

$$
\begin{aligned}
S E R_{n}^{W N C} & =\sum_{S_{n}=0}^{2^{(N-1)}-1} F\left(\left(1+\frac{b E_{s, n n}^{W N C} \sigma_{0 n}^{2}}{N_{0} \sin ^{2} \theta}\right) \times\right. \\
& \left.\prod_{\substack{r=1 \\
r \neq n}}^{N}\left(1+\frac{b E_{s, r n}^{W N C} \sigma_{0 r}^{2} \beta_{r n}}{N_{0} \epsilon_{n} \sin ^{2} \theta}\right)\right) \prod_{\substack{r=1 \\
r \neq n}}^{N} G\left(\beta_{r n}\right),
\end{aligned}
$$

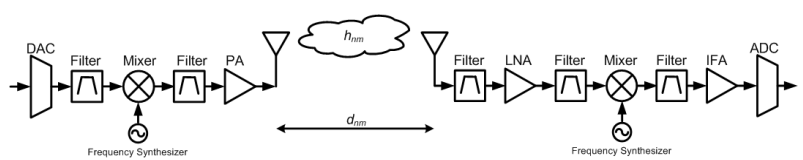

Fig. 2. Transmitter and receiver chains.

where $E_{s, n n}^{W N C}$ and $E_{s, r n}^{W N C}$ (for $r \neq n$ ) are, respectively, the energy per symbol allocated at the source node $U_{n}$ and at the relay node $U_{r}, \epsilon_{n}$ is the interference impact due to the crosscorrelations in forming the unique signal at a relay node, and $\beta_{r n} \in\{0,1\}$ for $r \neq n$ represents a detection state at $U_{r}$. When $U_{r}$ detects $U_{n}$ 's information correctly, $\beta_{r n}=1$; otherwise, $\beta_{r n}=0$. All $\beta_{r n}$ 's form the decimal number $S_{n}$ in (4), i.e., $S_{n}=\left[\beta_{1 n} \ldots \beta_{r n} \ldots \beta_{N n}\right]_{2}$ that represents one of $2^{N-1}$ network detection states associated with information from $U_{n}$. In (4), we also have

$$
G\left(\beta_{r n}\right)=\left\{\begin{array}{ll}
1-S E R_{r n} & \text { if } \beta_{r n}=1 \\
S E R_{r n} & \text { if } \beta_{r n}=0
\end{array},\right.
$$

where

$$
S E R_{r n}=F\left(1+\frac{b E_{s, n n}^{W N C} \sigma_{r n}^{2}}{N_{0} \sin ^{2} \theta}\right)
$$

is the SER in detecting $U_{n}$ 's information at $U_{r}$. Note that the total symbol energy associated with $U_{n}$ 's information is $E_{s, n}^{W N C}=\sum_{r=1}^{N} E_{s, r n}^{W N C}$.

Given a bit rate $R_{b}$ and a constellation size $\mathcal{M}$, the required transmit power can be expressed as

$$
P_{s, n}=E_{s, n}\left(R_{b} / \log _{2}(\mathcal{M})\right)
$$

where $E_{s, n}$ is the symbol energy in (2) and (4) for DTX and WNC, respectively.

\subsection{Power Consumption in DTX Networks}

Figure 2 illustrates the transmitter and receiver chains of single-antenna systems. As modeled in [7], power consumption includes two major parts, the power consumption by the $\mathrm{PA}$ and the power consumption of other RF components. In this work, we neglect the power consumption of the baseband signal processing blocks such as those to perform forward error correction (FEC) and modulation. Nevertheless, the power consumption for these blocks [8] can be incorporated into the model in future work.

In DTX networks, a user node $U_{n}$ only transmits its own information. Thus its PA power consumption is

$$
P_{P A, n}^{D T X}=\frac{\xi}{\eta} P_{s, n}^{D T X}
$$

where $\xi$ is the PAPR, $\eta$ is the PA efficiency, and $P_{s, n}^{D T X}$ is the required transmit power in DTX to convey the information over the transmission medium and is computed following (7). $U_{n}$ also incurs the transmitter processing power due to the power consumption at the transmitter RF components. The transmitter power consumption can be given as

$$
P_{T P, n}^{D T X} \approx P_{D A C}+P_{f i l t}+P_{m i x}+P_{s y n},
$$


where $P_{D A C}, P_{f i l t}, P_{m i x}$, and $P_{s y n}$ are the power consumption at the digital-to-analog converter (DAC), the transmit filters, the mixer, and the frequency synthesizer, respectively. $P_{\text {filt }}, P_{\text {mix }}$, and $P_{\text {syn }}$ can be modeled as constants [7] while $P_{D A C}$ can be approximated as [7]

$$
\begin{aligned}
P_{D A C} \approx & \left(\frac{1}{2} V_{d d} I_{0}\left(2^{n_{1}}-1\right)+\right. \\
& \left.n_{1} C_{p}\left(2 B+f_{c o r}\right) V_{d d}^{2}\right),
\end{aligned}
$$

where $V_{d d}$ and $I_{0}$ are the voltage and current supplies, $C_{p}$ is the parasitic capacitance, $n_{1}$ is the number of bits in the DAC, $B=R_{b} / \log _{2}(\mathcal{M})$ is the symbol bandwidth, and $f_{\text {cor }}$ is the corner frequency. The total power consumption for $U_{n}$ in DTX is

$$
P_{n}^{D T X}=P_{P A, n}^{D T X}+P_{T P, n}^{D T X} .
$$

The base node $U_{0}$ 's function is only receiving signals from the user nodes. Thus the power consumption at $U_{0}$ is also the receiver processing power consumption and can be given as

$P_{0}^{D T X} \approx N\left(P_{L N A}+P_{f i l r}+P_{m i x}+P_{I F A}+P_{A D C}+P_{s y n}\right)$,

where $P_{L N A}, P_{\text {filr }}, P_{m i x}, P_{s y n}, P_{I F A}$, and $P_{A D C}$ are the power consumption at low-noise amplifier (LNA), the receive filters, the mixer, the frequency synthesizer, the intermediatefrequency amplifier (IFA), and the analog-to-digital converter (ADC), respectively. The factor of $N$ in (12) is due to the fact that $U_{0}$ receives $N$ times in DTX networks. Like the transmitter RF chain, $P_{L N A}, P_{f i l r}, P_{m i x}, P_{s y n}$, and $P_{I F A}$ can be modeled as constants [7] while $P_{A D C}$ can be approximated as [7]

$$
P_{A D C} \approx \frac{3 V_{d d}^{2} L_{\min }\left(2 B+f_{c o r}\right)}{10^{-0.1525 n_{2}+4.838}},
$$

where $L_{\min }$ is the minimum channel length in the complementary metal-oxide-semiconductor (CMOS) technology and $n_{2}$ is the number of bits in the ADC.

\subsection{Power Consumption in WNC Networks}

Since all nodes in WNC networks are assumed to have a single antenna, the transmitter and receiver chain follows Figure 2 . In WNC networks, user node $U_{n}$ transmits its own information in the STP and relays other nodes' information in the RTP. We assume equal power consumption strategy, which allocates one half of the required transmit power at the source node and equally divides the other half at the $(N-1)$ relay nodes. Thus the PA power consumption of user node $U_{n}$ is

$$
P_{P A, n}^{W N C}=\frac{\xi}{\eta}\left(\frac{1}{2} P_{s, n}^{W N C}+\sum_{\substack{r=1 \\ r \neq n}}^{N} \frac{1}{2(N-1)} P_{s, r}^{W N C}\right),
$$

where $\xi$ is the PAPR, $\eta$ is the PA efficiency, and $P_{s, n}^{W N C}$ and $P_{s, r}^{W N C}$ follow (7). In addition, $U_{n}$ incurs transmitter processing power consumption, which can be given as

$$
P_{T P, n}^{W N C}=P_{T P, S T P}^{W N C}+P_{T P, R T P}^{W N C},
$$

where $P_{T P, S T P}^{W N C}$ and $P_{T P, R T P}^{W N C}$ are the power consumption at the transmitter RF components in the STP and the RTP, respectively. $P_{T P, S T P}^{W N C}$ and $P_{T P, R T P}^{W N C}$ follows (9), in which $P_{D A C}$ takes the form of (10) in the STP and

$$
\begin{aligned}
& P_{D A C} \approx\left(\frac{1}{2} V_{d d} I_{0}\left(2^{n_{1}}-1\right)+\right. \\
& \left.n_{1} C_{p}\left(2(N-1) B+f_{c o r}\right) V_{d d}^{2}\right)
\end{aligned}
$$

in RTP. Note that the factor of $(N-1)$ in (16) is due to use of FDMA or CDMA techniques to relay overheard information in WNC. In the STP, $U_{n}$ also consumes power in reception of signals from other user nodes. The power consumption can be expressed as

$$
\begin{array}{r}
P_{R P, n}^{W N C} \approx(N-1)\left(P_{L N A}+P_{f i l r}+P_{\text {mix }}+\right. \\
\left.P_{I F A}+P_{A D C}+P_{\text {syn }}\right),
\end{array}
$$

where $P_{A D C}$ follows (13) and the power consumption of the remaining components can be modeled as constants. Note that the factor of $(N-1)$ in (17) accounts for the $(N-1)$ receptions at $U_{n}$ in STP. In summary, the power consumption at user node $U_{n}$ in WNC is

$$
P_{n}^{W N C}=P_{P A, n}^{W N C}+P_{T P, n}^{W N C}+P_{R P, n}^{W N C} .
$$

The base node $U_{0}$ in WNC receives signals in both phases. The receiver processing power consumption can be expressed as

$$
P_{0}^{W N C}=P_{R P, S T P}^{W N C}+P_{R P, R T P}^{W N C},
$$

where $P_{R P, S T P}^{W N C}$ and $P_{R P, R T P}^{W N C}$ are the receiver power consumption in the STP and RTP, respectively. $P_{R P, S T P}^{W N C}$ and $P_{R P, R T P}^{W N C}$ follow (12), in which $P_{A D C}$ takes the form of (13) for the STP and

$$
P_{A D C} \approx \frac{3 V_{d d}^{2} L_{\min }\left(2(N-1) B+f_{c o r}\right)}{10^{-0.1525 n_{2}+4.838}}
$$

in the RTP. Again, the factor of $(N-1)$ is due to the use of FDMA or CDMA technique in the relaying signals in WNC.

\subsection{Simulations}

We perform computer simulations of the power consumption in DTX and WNC networks to realize the power saving of WNC over DTX. In this work, a power saving is defined as a ratio of the power consumption in DTX over that in WNC. We consider two power savings: network power saving, denoted as $S_{n e t}$, and individual power saving, denoted as $S_{i n d}$. We define

$$
S_{n e t} \triangleq \frac{\sum_{n=0}^{N} P_{n}^{D T X}}{\sum_{n=0}^{N} P_{n}^{W N C}}, \quad S_{\text {ind }} \triangleq \frac{P_{n}^{D T X}}{P_{n}^{W N C}} \text { (times). }
$$

Table 1 lists the parameters used in this simulation. We consider corner networks, in which the base node locates at $(0,0)$ and $N$ user nodes are uniformly distributed in a square area $\mathcal{A}=[0, D]^{2}$, where $D$ measured in meters denotes the network dimension. Without loss of generality, the user nodes are numbered in decreasing order of their distance to the base node. In this manner, $U_{1}$ and $U_{N}$ are the farthest and the closest to $U_{0}$, respectively. The number of user nodes is fixed with $N=7$ while the network dimension takes different values. For each network dimension, 50 network realizations were generated. 
Table 1. Simulation parameters

\begin{tabular}{|l|l|l|}
\hline Transmission parameters & RF parameters [7] & $P_{D A C}$ and $P_{A D C}$ parameters [7] \\
\hline$f_{c}=400 \mathrm{MHz}$ & $\xi=4 \mathrm{~dB}(\mathrm{QPSK})$ & $V_{d d}=3 \mathrm{~V}$ \\
$\alpha=3$ & $\eta=0.35$ & $I_{0}=10 \mu \mathrm{A}$ \\
$G_{t}=G_{r}=3 \mathrm{dBi}$ & $N_{f}=7$ & $C_{p}=1 \mathrm{pF}$ \\
$N_{0}=-174 \mathrm{dBm} / \mathrm{Hz}$ & $P_{\text {mix }}=30.0 \mathrm{~mW}$ & $L_{\text {min }}=0.5 \mu \mathrm{m}$ \\
$R_{b}=10 \mathrm{Kbps}$ & $P_{\text {filt }}=P_{\text {filr }}=2.5 \mathrm{~mW}$ & $f_{\text {cor }}=1 \mathrm{MHz}$ \\
$\mathcal{M}=4(\mathrm{QPSK})$ & $P_{L N A}=20 \mathrm{~mW}$ & $n_{1}=16(16-\mathrm{bit} \mathrm{DAC})$ \\
$S E R_{0}=2 \mathrm{e}-3$ & $P_{I F A}=3 \mathrm{~mW}$ & $n_{2}=14(14-\mathrm{bit} \mathrm{ADC})$ \\
(equivalently $\left.B E R_{0}=1 \mathrm{e}-3\right)$ & $P_{\text {syn }}=50.0 \mathrm{~mW}$ & \\
\hline
\end{tabular}

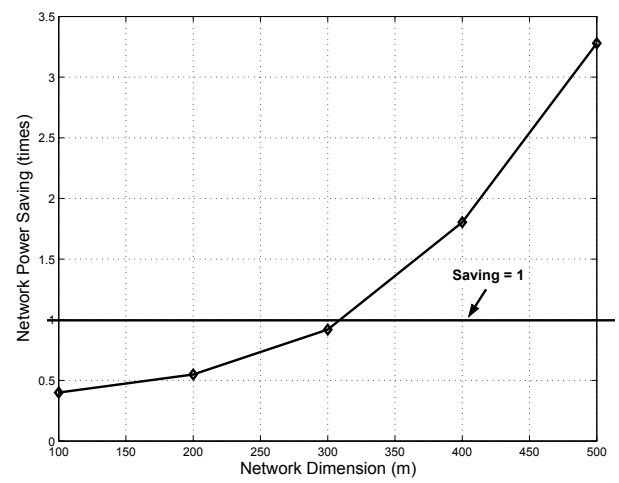

Fig. 3. Average network power saving of WNC over DTX for $N=7$.

Figure 3 presents the average network power saving of WNC over DTX. As shown in the figure, WNC does not always lead to network power saving over DTX; the power saving is only realized for large network dimensions with $D>$ $300 \mathrm{~m}$. The cooperation among nodes in WNC leads to transmit power saving; the larger the transmission distance is the larger the transmit power saving of WNC over DTX. However, the cooperation also incurs additional processing power due to the reception and retransmission at the relay nodes. For small network dimensions, the saving in transmit power cannot compensate the additional processing power needed for cooperation. As a result, WNC consumes more power than DTX for these network dimensions.

Figure 4 presents the average power saving of individual nodes when participating in WNC over that in DTX for networks generated in Figure 3. An interesting observation is that for network dimensions associated with network power saving of WNC over DTX, not all user nodes have individual power saving. For example, $U_{7}$ in $\mathrm{WNC}$ incurs more power consumption over DTX for $D=400 \mathrm{~m}$ and $500 \mathrm{~m}$. It is the closest node to the base node and thus requires much less transmit power in DTX. When participating in WNC, the transmit power saving is not large enough to compensate the additional processing power required in cooperation. As a result, it consumes more power in WNC than in DTX.

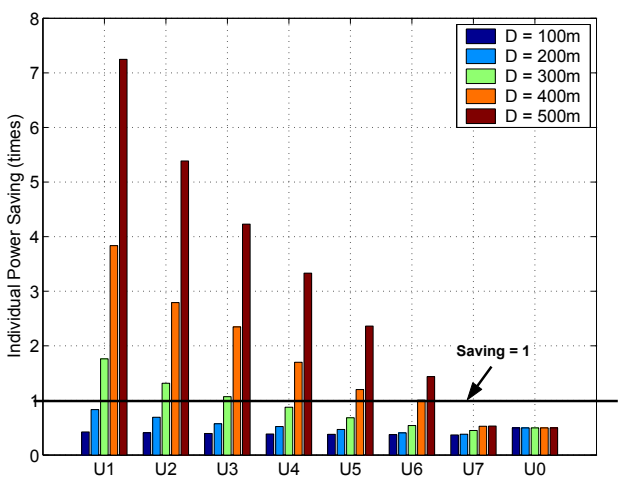

Fig. 4. Average individual power saving of WNC over DTX for $N=7$.

\section{COALITION FORMATION GAMES FOR ENERGY EFFICIENT WNC}

\subsection{Merge Process for WNC Networks}

Coalition formation games have been applied in economics and political science. Recently, they are also used to analyze performance of communication networks [3]. Fundamentally, a coalition formation game consists of a three-tuple $(\mathcal{P}, v, \mathbf{x})$ [3], where $\mathcal{P}=\left\{U_{1}, U_{2}, \ldots, U_{N}\right\}$ is the set of user nodes who seek to form cooperative groups (or coalitions) to improve their performance, $v$ is a utility function that defines how the game would play, and $\mathbf{x}$ is a vector representing the payoffs the members would receive from the value $v$. We define the utility function and individual payoff for WNC networks as

$$
\begin{gathered}
v(C)=\sum_{U_{n} \in C}\left(P_{P A, n}^{D T X}-P_{P A, n}^{W N C}\right)- \\
\sum_{U_{n} \in C}\left(\left(P_{T P, n}^{W N C}+P_{R P, n}^{W N C}\right)-P_{T P, n}^{D T X}\right),
\end{gathered}
$$

and

$$
\begin{gathered}
x_{n}(C)=\left(P_{P A, n}^{D T X}-P_{P A, n}^{W N C}\right)- \\
\left(\left(P_{T P, n}^{W N C}+P_{R P, n}^{W N C}\right)-P_{T P, n}^{D T X}\right),
\end{gathered}
$$

respectively, where $C$ denotes a cooperative group with members $U_{n}$ 's. Note when $C=\left\{U_{n}\right\}$, a single-member coalition, the power terms associated with WNC converge to those associated with DTX. In that case $v(C)=x_{n}(C)=0$. In (22), the first summation is the transmission power saving while the second summation is the additional processing power. They represent the gain and the cost, respectively, in cooperation. As the size of the cooperative group increases, both 
the gain and the cost also increase. The additional processing power linearly increases with the size of the cooperative group. However, the transmit power saving in cooperation gradually diminishes due to the nature of incremental diversity that causes the relative transmit power saving to reduce [9]. At some point, user nodes can no longer be added to the cooperative group. This prevents WNC networks from forming a grand coalition [3]. The problem now is to find the optimal coalition structures. In [4], merge and split rules are proposed to form cooperative groups and proved to converge to a unique solution with arbitrary merge and split iterations. However, the complexity of exchanging information in wireless networks is too high, especially with large numbers of user nodes $N$. To make the problem traceable, we propose a heuristic approach to form the cooperative groups in WNC.

As proposed in [4], a merge between two cooperative groups $C_{1}$ and $C_{2}$ happens if it increases the value of the utility function, i.e.

$$
v\left(C_{1} \cup C_{2}\right)>v\left(C_{1}\right)+v\left(C_{2}\right) \text { for } C_{1} \cap C_{2}=\emptyset .
$$

This condition is to ensure that the network power saving of the merged group would be achieved. To avoid additional power burden to individual nodes in cooperation, we impose the second condition as

$$
x_{n}\left(C_{1} \cup C_{2}\right) \geq x_{n}\left(C_{i}\right) \forall n,
$$

for $i=1$ or 2 , the index of the cooperative group that $U_{i}$ belongs to before merging $C_{1}$ and $C_{2}$.

Given the merge conditions in (24) and (25), we propose a TDMA-based merge algorithm that ensures the information exchange among the members occur orderly and efficiently. The merge process includes three phases: transmission request, merge process, and WNC transmission.

In the first phase, the $N$ user nodes send a request-to-send (RTS) signal to the base node $U_{0}$. The user nodes are set to use a maximum transmission power so that other user nodes can estimate the inter-user channel variances. After receiving the RTS, $U_{0}$ broadcasts a WNC transmission schedule as shown in Figure 5(a), which includes $2 N$ time slots for the STP and RTP of WNC transmissions. Based on the broadcasting signal from $U_{0}$, the user nodes can also estimate the user-base channel variances. This information will be used later in the merge process. The WNC transmission schedule also indicates the transmission order, starting from the farthest to the closest user nodes. Without loss of generality, we can number the user nodes in decreasing order of their distance to the base node. In this manner, $U_{1}$ and $U_{N}$ are the farthest and the closest to $U_{0}$, respectively.

Based on the order in the WNC transmission schedule, starting from $U_{1}$ down to $U_{N}$, the merge process takes place to form cooperative groups. In this manner, disadvantageous nodes in terms of transmit power consumption will receive more assistance to lower their power burden. In the merge process, each node is allowed a maximum number of attempts, denoted as Max, which is used to control the overhead in forming cooperative groups. The merge schedule for the merge process is shown in Figure 5(b), which is TDMA-based. Note that the time slots in the merge schedule are much shorter than those in the WNC transmission schedule. Assume at present that we attempt to merge at user node

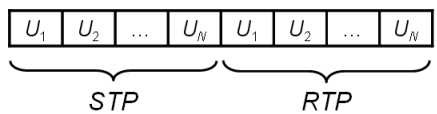

(a)

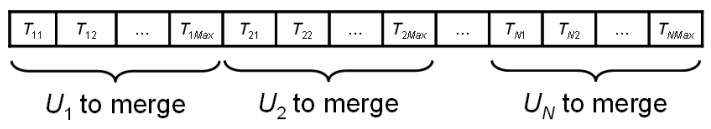

(b)

Fig. 5. (a) WNC transmission schedule and (b) Merge schedule.

$U_{n}$. If $U_{n}$ already belongs to some cooperative groups due to previous merges at other user nodes, then $U_{n}$ remains silent during its assigned $T_{n 1}, T_{n 2}, \ldots, T_{n M a x}$ time slots. Otherwise, $U_{n}$ begins to merge with other remaining nodes in the network. In this case, $U_{n}$ is called the coalition head and its index $n$ is used for the coalition index.

The merge at $U_{n}$ happens as the following. At the beginning, $U_{n}$ broadcasts its user-base channel variance to its neighbors. Assume at present that $U_{n}$ attempts to merge with $U_{r}$, its closest non-member neighbor. $U_{n}$ first sends the indices of its members to $U_{r}$ and requests for a merge. $U_{r}$ agrees to merge if the following three conditions are satisfied. Firstly, $U_{r}$ does not belong to any cooperative groups. Secondly, it has inter-user and user-base channel variances for all coalition members. This condition is to ensure full diversity for all user nodes in the cooperative group. Note that $U_{r}$ obtains the inter-user channel variances during the transmission request phase and the user-base channel variances in the broadcasting signals from other members who already join the cooperative group. Lastly, conditions in (24) and (25), computed by $U_{r}$, are satisfied. When $U_{r}$ agrees to merge, it acknowledges the merge back to the coalition members, sends the transmit power requirements for each nodes in the cooperative group, and broadcasts its user-base channel variance. User nodes in range of $U_{r}$ 's transmission can record $U_{r}$ 's user-base channel variance for later computation when it is requested to merge. $U_{n}$ then repeats its attempts to the next closest non-member node until there is no node available to merge or when $U_{n}$ expires all its attempts.

After the cooperative groups have formed, WNC transmissions take place. Each user node takes turn to transmit based on $U_{0}$ 's WNC transmission schedule in Figure 5(a). A user node only decodes and relays information for its members. The base station detects information of user nodes based on the cooperative groups.

\subsection{Simulations}

We perform computer simulations to validate the proposed merge process for WNC networks. The performance metrics of network power saving and individual power saving defined in (21) are used. For the simulation setup, the simulation parameters are listed in Table 1 . We consider a center network, in which the base node locates at $(0,0)$ and $N$ user nodes, with $N=30$, are uniformly distributed in a square area $\mathcal{A}=[-500 \mathrm{~m}, 500 \mathrm{~m}]^{2}$. The user nodes are numbered in decreasing order of their distance to the base node with $U_{1}$ and $U_{N}$ being the farthest and the closest to $U_{0}$, respectively. In addition, we assume $\operatorname{Max}=2$. 


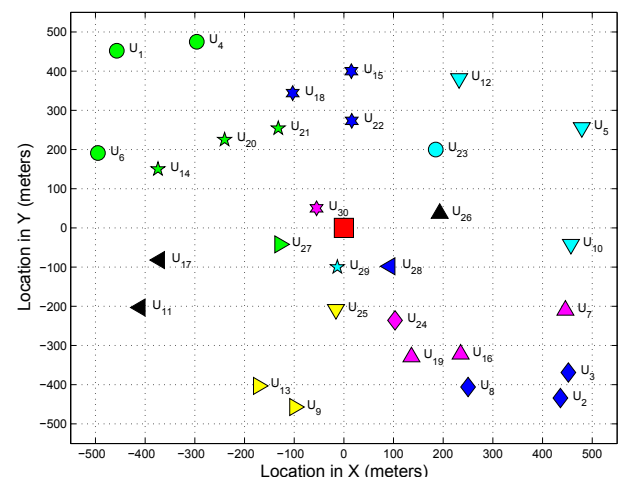

Fig. 6. Coalition structure (shape-and-color coded) for a WNC network of $N=30$ and $\operatorname{Max}=2$. The associated network power saving is 3.4 times.

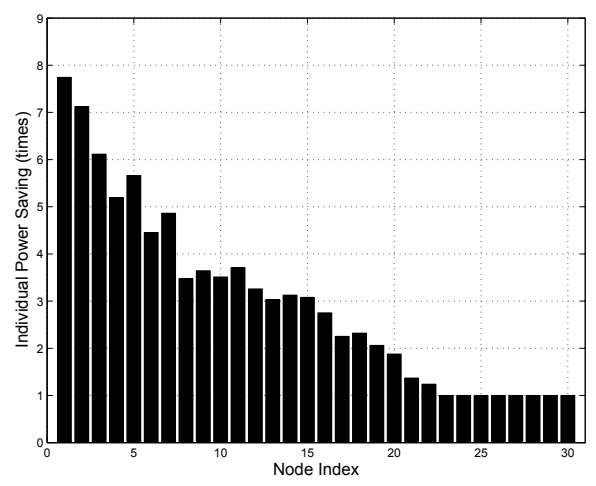

Fig. 7. Individual power savings for WNC network shown in Figure 6.

Figure 6 shows the coalition structure generated by the proposed merge process. In the figure, the cooperative groups are shape-and-color coded. Overall, the associated network power saving is 3.4 times. From the figure, some of user nodes, for example, $\left\{U_{25}\right\},\left\{U_{26}\right\}$, and $\left\{U_{27}\right\}$, stand by themselves, creating single-member coalitions. These nodes are in locations close to the base node and do not have large transmit power saving to compensate the additional processing power when cooperating with other nodes. The figure also shows that some cooperative groups, for example $\left\{U_{9}, U_{13}\right\}$ and $\left\{U_{11}, U_{17}\right\}$, do not have the full coalition size (i.e. $\left.M a x+1\right)$. Although these cooperative groups welcome additional members since that would help reducing their power consumption, other nodes may not find the benefits to join due to the additional power burden to themselves, and thus larger cooperative groups could not be formed.

Figure 7 shows the individual power saving for the coalition structure in Figure 6. The individual power savings are all greater or equal to one for all individual user nodes. This clearly shows the cooperation does not cause additional power burden to individual nodes, as expected.

Figure 8 shows the power distribution among the user nodes in WNC and DTX networks shown in Figure 6. The figure plots the power consumption of individual nodes versus the node index. From the figure, nodes with lower indices, which are associated with larger transmission distances, have higher power consumption in DTX network. This is due to the

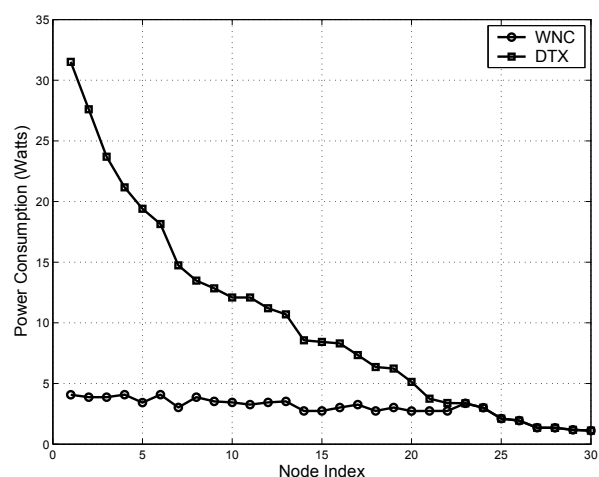

Fig. 8. Power distribution among user nodes in WNC and DTX networks shown in Figure 6.

dependency of transmit power consumption on the transmission distance. On the other hand, the power consumption of individual nodes in WNC network is comparable. This illustrates another advantage of using WNC over DTX; the even power distribution helps improving the network lifetime.

\section{CONCLUSIONS}

In this work, we examined the power consumption in WNC and DTX networks and proposed a TDMA-based merge process to ensure energy efficiency WNC networks. The merge process is based on coalition formation games to orderly and efficiently form the cooperative groups in WNC networks. A node is merged into a cooperative group if the merge leads to power saving for the group without causing additional power burden to the individual members. Simulation is provided to corroborate the energy efficient WNC.

Approved for public release. Distribution is unlimited.

\section{REFERENCES}

[1] K. J. R. Liu, A. K. Sadek, W. Su, and A. Kwasinski, Cooperative Communications and Networking. Cambridge, UK: Cambridge University Press, 2008.

[2] H. Q. Lai and K. J. R. Liu, "Space-time network coding," IEEE Trans. Signal Proces., vol. To appear, Apr. 2011.

[3] W. Saad, Z. Han, and M. Debbah, "Coalitional game theory for communication networks,” IEEE Signal Process. Mag., pp. 77-97, Sep. 2009.

[4] K. Apt and A. Witzel, "A generic approach to coalition formation," Proc. Int. Workshop Computational Social Choice (COMSOC), Dec. 2006.

[5] W. Saad, Z. Han, M. Debbah, and A. Hjorungnes, "A distributed coalition formation framework for fair user cooperation in wireless networks," IEEE Trans. Wireless Commun., vol. 8, no. 9, pp. 4580-4593, Sep. 2009.

[6] J. G. Proakis, Digital Communications. New York, USA: McGraw-Hill, 4th ed., 2001.

[7] S. Cui, A. J. Goldsmith, and A. Bahai, "Energy-constraint modulation optimization," IEEE Trans. Wireless Commun., vol. 4, no. 5, pp. 23492360, Sep. 2005.

[8] H. S. Kim and B. Daneshrad, "Energy-aware link adaptation for MIMOOFDM based wireless communication," Proc. IEEE MILCOM, pp. 1-7, Nov. 2008.

[9] H. Q. Lai, A. Ibrahim, and K. J. R. Liu, "Wireless network cast: Location-aware cooperative communications with linear network coding," IEEE Trans. Wireless Commun., vol. 8, no. 7, pp. 3844-3854, Jul. 2009. 